Pacific Journal of Mathematics

EXPOSED POINTS OF LEFT INVARIANT MEANS 


\title{
EXPOSED POINTS OF LEFT INVARIANT MEANS
}

\author{
ZHUOCHENG YANG
}

\begin{abstract}
If $S$ is a left amenable semigroup, let $M L(S)$ be the set of left invariant means on $m(S)$, the space of bounded real-valued functions on $S$. We prove in this paper that a left invariant mean on $m(S)$ is an exposed point of $M L(S)$ if and only if it is the arithmetic average on a minimal finite left ideal of $S$. In particular, $M L(S)$ has no exposed point when $S$ is an infinite group. We also prove that if $M L(S)$ has an exposed point, then it is the $w^{*}$-closed convex hull of all its exposed points. This gives another proof of the Granirer-Klawe theorem on the dimension of $M L(S)$.
\end{abstract}

1. Introduction. For an arbitrary set $X$, let $m(X)$ be the Banach space of bounded real-valued functions on $X$ with the supremum norm. An element $\mu \in m(X)^{*}$ is called a mean on $m(X)$ if $\mu$ is positive and $\|\mu\|=1$. A finite mean on $X$ is a positive element $\mu \in l^{1}(X)$ with $\|\mu\|_{1}=1$, and such that the support of $\mu$, the set $\{x \in X \mid \mu(x)>0\}$, is finite. Any finite mean, considered as an element of $m(X)^{*}$, is a mean. And the set of all finite means is $w^{*}$-dense in the set of all means on $X$ (see Day [2]).

Let $S$ be a semigroup. A mean $\mu$ on $m(S)$ is left invariant if $\mu(f)=\mu\left(l_{s} f\right)$ for all $f \in m(S)$ and $s \in S$, where $l_{s} f \in m(S)$ is defined by $\left(l_{s} f\right)(t)=f(s t), t \in S$. When $m(S)$ has a left invariant mean, we say $S$ is left amenable, and denote by $M L(S)$ the set of all left invariant means on $m(S)$. $M L(S)$ is convex and $w^{*}$-compact in $m(S)^{*}$ (cf. [2]). If $s \in S$ and $A \subset S$, it is easy to see that $\mu\left(\chi_{s A}\right) \geq \mu\left(\chi_{A}\right)$ for any $\mu \in$ $M L(S)$.

For a mean $\mu$ on $m(S)$ and $s \in S$, we define $s \cdot \mu \in m(S)^{*}$ by $(s \cdot \mu) f=\mu\left(l_{s} f\right), f \in m(S) . s \cdot \mu$ is also a mean on $m(S)$, and $(s t) \cdot \mu=$ $s \cdot(t \cdot \mu)$ for $s, t \in S$. If $\left\{\mu_{\lambda}\right\}$ is a net of means on $m(S)$, we say that $\left\{\mu_{\lambda}\right\}$ is $w^{*}$-convergent to left invariance if the net $\left\{s \cdot \mu_{\lambda}-\mu_{\lambda}\right\}$ is $w^{*}$-convergent to 0 for each $s \in S$. Day [2] proved that $S$ is left amenable if and only if there exists a net of finite means $w^{*}$-convergent to left invariance.

When $S$ is left amenable, $M L(S)$, as a $w^{*}$-compact convex set, is the $w^{*}$-closed convex hull of all its extreme points. It is natural to ask how many exposed points (with respect to the $w^{*}$-topology) $M L(S)$ has. Chou 
[1] proved that if $G$ is a countable infinite amenable group, then $M L(G)$ has no exposed points. Later, Granirer [4] studied intensively the existence of exposed points of subsets of $M L(S)$ for a countable left amenable semigroup $S$. In particular, he proved [4, Cor. 4.1] that if $S$ is a countable left amenable semigroup, then $M L(S)$ has exposed points if and only if $S$ has finite left ideals. In this paper we characterize the exposed points of $M L(S)$ for an arbitrary left amenable semigroup $S$ as the arithmetic averages on minimal finite left ideals. Thus we are abel to prove Chou and Granirer's results without the countability condition. We also prove that if $M L(S)$ has an exposed point, then it is the $w^{*}$-closed convex hull of all its exposed points. This gives another proof of the Granirer-Klawe Theorem on the dimension of $M L(S)$ (see [5]).

This paper will form part of my thesis under the supervision of Professor Anthony T. Lau. The author is most indebted to Professor Lau for his valuable suggestions and encouragement.

2. Some lemmas. In this section we are going to prove some lemmas which are used to obtain the main results. They are also of independent interest.

For convenience we write $\mu(A)$ for $\mu\left(\chi_{A}\right)$ when $\mu$ is a mean and $A$ is a subset of the underlying set or semigroup.

LemMa 2.1. Let $X$ be an infinite set, $\left\{\mu_{\lambda}\right\}_{\lambda \in \Lambda}$ a net of finite means $w^{*}$-convergent to a mean $\mu$. Let $\kappa$ be an infinite cardinal. If for each subset $A$ of $X, \mu(A)=0$ whenever $|A|<\kappa$, then $|\Lambda|>\kappa$.

Proof. Suppose $|\Lambda|=\kappa$. We are going to construct a function $f \in$ $m(X)$ such that $\mu_{\lambda}(f)$ diverges.

Well order $\Lambda$ as $\left\{\lambda_{\alpha}\right\}_{\alpha<\kappa}$. We define $f$ by transfinite induction.

Let $\alpha<\kappa$ be an ordinal. Suppose we have defined for each $\beta<\alpha$ a function $f_{\beta}$ with range $\{0,1\}$ on a subset $A_{\beta}$ of $X$, satisfying

(1) If $\beta$ is finite, then $A_{\beta}$ is finite. If $\beta$ is infinite, then $\left|A_{\beta}\right| \leq|\beta|$.

(2) $\beta_{1}<\beta_{2}<\alpha \Rightarrow A_{\beta_{1}} \subset A_{\beta_{2}}$ and $f_{\beta_{2}} \uparrow A_{\beta_{1}}=f_{\beta_{1}}$.

(3) If $\beta<\alpha$, then there exists $\lambda^{\prime}, \lambda^{\prime \prime}>\lambda_{\beta}$ in $\Lambda$, such that the supports of $\mu_{\lambda^{\prime}}$ and $\mu_{\lambda^{\prime \prime}}$ are contained in $A_{\beta}$, and $\mu_{\lambda^{\prime}}\left(f_{\beta}\right)<1 / 4, \mu_{\lambda^{\prime \prime}}\left(f_{\beta}\right)>$ $3 / 4$.

If $\alpha$ is finite, then $\bigcup_{\beta<\alpha} A_{\beta}$ is finite. If $\alpha$ is infinite, then $\left|\bigcup_{\beta<\alpha} A_{\beta}\right| \leq|\alpha|^{2}$ $=|\alpha|$. In both cases $\mu\left(\cup_{\beta<\alpha} A_{\beta}\right)=0 . \mu_{\lambda} \stackrel{w^{*}}{\rightarrow} \mu$ implies that there exists 
$\lambda^{\prime}>\lambda_{\alpha}$ in $\Lambda$, such that $\mu_{\lambda^{\prime}}\left(X \backslash \bigcup_{\beta<\alpha} A_{\beta}\right)>3 / 4$. Also since $\mid \bigcup_{\beta<\alpha} A_{\beta}$

$\cup \operatorname{supp} \mu_{\lambda^{\prime}} \mid<\kappa$, there exists $\lambda^{\prime \prime}>\lambda_{\alpha}$ in $\Lambda$ such that

$$
\mu_{\lambda^{\prime \prime}}\left(X \backslash\left(\bigcup_{\beta<\alpha} A_{\beta} \cup \operatorname{supp} \mu_{\lambda^{\prime}}\right)\right)>3 / 4 \text {. }
$$

Let $A_{\alpha}=\cup_{\beta<\alpha} A_{\beta} \cup \operatorname{supp} \mu_{\lambda^{\prime}} \cup \operatorname{supp} \mu_{\lambda^{\prime \prime}}$, and define

$$
f_{\alpha}(s)= \begin{cases}f_{\beta}(s), & \text { if } s \in A_{\beta} \text { for some } \beta<\alpha, \\ 0, & \text { if } s \in \operatorname{supp} \mu_{\lambda^{\prime}} \backslash \bigcup_{\beta<\alpha} A_{\beta}, \\ 1, & \text { if } s \in A_{\alpha} \backslash\left(\bigcup_{\beta<\alpha} A_{\beta} \cup \operatorname{supp} \mu_{\lambda^{\prime}}\right) .\end{cases}
$$

It is easy to see that $A_{\alpha}$ and $f_{\alpha}$ satisfy conditions (1)-(3).

Now let $f=f_{\alpha}$ on $A_{\alpha}, \alpha<\kappa$, and $f=0$ on $X \backslash \bigcup_{\beta<\alpha} A_{\alpha}$. Then $\mu_{\lambda}(f)$ diverges. In fact

$$
\liminf \mu_{\lambda}(f) \leq \frac{1}{4}<\frac{3}{4} \leq \lim \sup \mu_{\lambda}(f) .
$$

COROLlary 2.2. Let $S$ be an infinite left amenable right cancellative semigroup, $\left\{\mu_{\lambda}\right\}_{\lambda \in \Lambda}$ a net of finite means $w^{*}$-convergent to a left invariant mean $\mu$. Then $|\Lambda|>|S|$.

Proof. Let $A \subset S$ be such that $|A|<|S|$. Then it is not difficult to see that $\mu(A)=0$. A proof can be found in [5, Prop. 2.5].

LEMMA 2.3. Let $S$ be an infinite left amenable semigroup, $\mu$ an extreme point of $M L(S)$. Define the cardinal function $\kappa(\mu)=\min \{|A| \mid A \subset S$ and $\mu(A)=1\}$. If $\kappa(\mu)$ is infinite, then for each subset $B$ of $S,|B|<\kappa(\mu)$ implies $\mu(B)=0$.

Proof. Suppose the contrary that there is a set $B \subset S$ such that $|B|<\kappa(\mu)$ and $\mu(B)>0$.

If $B$ is finite, then there is an $s \in S$ with $\mu(\{s\})>0$. For any $t \in S$, $\mu(\{t s\}) \geq \mu(\{s\})$. So the left ideal $I=S s$ of $S$ is finite, and $0<\mu(I)<1$ since $\kappa(\mu)$ is infinite. For any $t \in S, \mu(t I) \geq \mu(I)$ and $t I \subset I$ give that $\mu(t I)=\mu(I)$ and $\mu(I \Delta t I)=0$.

Suppose $B$ is infinite. Let $x=\sup \{\mu(A)|A \subset S| A,|\leq| B \mid\}$. By taking countable union, we can get a subset $I$ of $S$ such that $|I|=|B|$ and $\mu(I)=x$. For any $t \in S, \mu(I) \leq \mu(t I) \leq \mu(t I \cup I) \leq x=\mu(I)$ since $|I \cup t I|=|I|=|B|$. So the equalities hold everywhere. Thus we also have 
$0<\mu(I)<1, \mu(I)=\mu(t I)$ and $\mu(I \Delta t I)=0$. Denote by $t^{-1}(t I)$ the set $\{s \in S \mid t s \in t I\}$. It is easy to see that $\mu\left(I \Delta t^{-1}(t I)\right)=0$, since $t^{-1}(t I) \supset I$ and $\mu\left(t^{-1}(t I)\right)=\mu(t I)=\mu(I)$.

Let $\mu_{1} \in m(S)^{*}$ be defined by

$$
\mu_{1}(f)=\frac{\mu\left(f \cdot \chi_{I}\right)}{\mu(I)}, \quad f \in m(S)
$$

Then $\mu_{1}$ is positive, $\left\|\mu_{1}\right\|=1$, and is left invariant:

$$
\begin{aligned}
\mu_{1}\left(l_{t} f\right) & =\frac{\mu\left(\left(l_{t} f\right) \cdot \chi_{I}\right)}{\mu(I)}=\frac{\mu\left(\left(l_{t} f\right) \cdot \chi_{t^{-1}(t I)}\right)}{\mu(I)} \\
& =\frac{\mu\left(l_{t}\left(f \cdot \chi_{t I}\right)\right)}{\mu(I)}=\frac{\mu\left(f \cdot \chi_{t I}\right)}{\mu(I)}=\frac{\mu\left(f \cdot \chi_{I}\right)}{\mu(I)}=\mu_{1}(f),
\end{aligned}
$$

since $\mu\left(I \Delta t^{-1}(t I)\right)=0$ and $\mu(I \Delta t I)=0$.

Let $\mu_{2}=\left(\mu-\mu(I) \cdot \mu_{1}\right) /(1-\mu(I))$. Then for $f \in m(S)$,

$$
\mu_{2}(f)=\frac{\mu(f)-\mu\left(f \cdot \chi_{I}\right)}{1-\mu(I)}=\frac{\mu\left(f \cdot \chi_{S \backslash I}\right)}{\mu(S \backslash I)} .
$$

So $\mu_{2}$ is also in $\operatorname{ML}(S)$, and

$$
\mu=\mu(I) \mu_{1}+(1-\mu(I)) \mu_{2}
$$

is not an extreme point.

LEMMA 2.4. Let $S$ be a left amenable semigroup, $\left\{\mu_{\alpha}\right\}_{\alpha \in \Gamma}$ a net of finite means $w^{*}$-convergent to left invariance. Then for any $\alpha \in \Gamma$, any $\varepsilon>0$, and any $s_{1}, \ldots, s_{n} \in S$, there exists a finite mean $\mu_{\alpha}^{\prime}$ which is a convex combination of elements $\mu_{\beta}, \beta>\alpha$, such that

$$
\left\|s_{i} \cdot \mu_{\alpha}^{\prime}-\mu_{\alpha}^{\prime}\right\|<\varepsilon, \quad i=1, \ldots, n .
$$

Proof. This was proved by Day [2, p. 524].

3. Main results. We are now ready to prove our main results concerning the exposed points of $M L(S)$. In all cases we shall consider only the $w^{*}$-topology on $M L(S)$.

THEOREM 3.1. Let $S$ be a left amenable semigroup, and $\mu$ an exposed point of $M L(S)$ (if any). Then $\mu$ is a finite mean. 
Proof. Let $\mu$ be an extreme point of $M L(S)$ and define $\kappa(\mu)$ as in Lemma 2.3. Suppose $\mu$ is not a finite mean. Then $\kappa(\mu)$ is infinite. Take $A \subset S$ so that $|A|=\kappa(\mu)$ and $\mu(A)=1$. Then for any $t \in S, \mu(t A \cap A)$ $=1$ since $\mu(t A)=1$. In particular $t A \cap A \neq \varnothing$; i.e., there exist $a, b \in A$ with $t a=b$. For fixed $a, b \in A$, let $S_{(a, b)}=\{t \in S \mid t a=b\}$. Then $\bigcup\left\{S_{(a, b)} \mid a, b \in A\right\}=S$.

Pick $f \in m(S)$ with $\|f\|=1$, and choose a net $\left\{\mu_{\alpha}\right\}_{\alpha \in \Gamma}$ of finite means $w^{*}$-convergent to $\mu$. Then $\left\{\mu_{\alpha}\right\}_{\alpha \in \Gamma}$ is $w^{*}$-convergent to left invariance and $\mu_{\alpha}(f) \rightarrow \mu(f)$.

Let $\Lambda$ be the set of all finite nonempty subsets of $A \times A$, directed by inclusion. Then $\Lambda$ is a directed set with $|\Lambda|=|A|=\kappa(\mu)$. Take $F=$ $\left\{\left(a_{i}, b_{i}\right) \mid i=1, \ldots, n\right\} \in \Lambda$. There exists $\alpha \in \Gamma$ such that for any $\beta>\alpha$, $\left|\mu_{\beta}(f)-\mu(f)\right|<1 / 2 n$. By the finite intersection property on right ideals (see [3]), $\bigcap_{i=1}^{n} a_{i} S \neq \varnothing$. Choose $a \in \bigcap_{i=1}^{n} a_{i} S$ ( $a$ is not necessarily in $A$ ), say $a=a_{i} s_{i}, i=1, \ldots, n$. By Lemma 2.4 , there exists a finite mean $\mu_{\alpha}^{\prime}$ which is a convex combination of elements $\mu_{\beta}, \beta>\alpha$, such that

$$
\left\|a \cdot \mu_{\alpha}^{\prime}-\mu_{\alpha}^{\prime}\right\|<\frac{1}{2 n}
$$

and

$$
\left\|\left(b_{i} s_{i}\right) \cdot \mu_{\alpha}^{\prime}-\mu_{\alpha}^{\prime}\right\|<\frac{1}{2 n}, \quad i=1, \ldots, n .
$$

For $t \in S_{\left(a_{i}, b_{i}\right)}$, we have

$$
\left\|t \cdot\left(a \cdot \mu_{\alpha}^{\prime}\right)-a \cdot \mu_{\alpha}^{\prime}\right\|=\left\|\left(b_{i} s_{i}\right) \cdot \mu_{\alpha}^{\prime}-a \cdot \mu_{\alpha}^{\prime}\right\|<\frac{1}{n} .
$$

Also

$$
\left|\left(a \cdot \mu_{\alpha}^{\prime}\right)(f)-\mu(f)\right|<\frac{1}{n} .
$$

Define $\mu_{F}=a \cdot \mu_{\alpha}^{\prime}$. Then the net $\left\{\mu_{F}\right\}_{F \in \Lambda} w^{*}$-converges to left invariance and $\lim \mu_{F}(f)=\mu(f)$. Since $\mu$ is an extreme point of $M L(S)$, by Lemma 2.3 , for any $B \subset S,|B|<\kappa(\mu)$ implies $\mu(B)=0$. By Lemma 2.1, $\left\{\mu_{F}\right\}_{F \in \Lambda}$ does not converge to $\mu$ since $|\Lambda|=|A|=\kappa(\mu)$. So it has a $w^{*}$-cluster point $\mu_{1}$ different from $\mu$. Since $\mu_{1} \in M L(S)$ and $\mu_{1}(f)=$ $\mu(f), \mu$ is not an exposed point of $M L(S)$.

For a finite nonempty set $I \subset S$, the arithmetic average on $I$ is the finite mean $\mu$ such that for each $a \in I, \mu(\{a\})=1 /|I|$.

THEOREM 3.2. Let $S$ be a left amenable semigroup. Then $\mu$ is an exposed point of $M L(S)$ if and only if it is the arithmetic average on a minimal finite left ideal of $S$. 
Proof. Let $I$ be a minimal finite left ideal of $S$. By a result of Mitchell ([6, pp. 256-257]), there exists $\mu \in M L(S)$ with $\mu(I)=1$. Since $I a=I$ for any $a \in I, I$ is right cancellative. Also $\mu(a I)=\mu(I)$ implies that $a I=I$ for any $a \in S$. Thus $I$ is left cancellative and in fact a finite group. $\mu$ as the unique invariant mean on $I$ is the arithmetic average on $I$. Let $f$ be the characteristic function of $I$. Then $\mu(f)=1$. For any $\mu_{1} \in M L(S)$, if $\mu_{1}(f)=\mu_{1}(I)=1$, then by the above argument, $\mu=\mu_{1}$. Thus $\mu$ is an exposed point of $M L(S)$. (Remark. Part of the proof is adopted from [3, Thm. 4.1].)

Suppose $\mu$ is an exposed point of $M L(S)$. Then $\mu$ is a finite mean by Theorem 3.1. Let $I$ be the support of $\mu$. For $a \in I$ and $t \in S, \mu(\{t a\}) \geq$ $\mu(\{a\})>0$, so $t a \in I$. Thus $I$ is a left ideal and it contains a minimal left ideal $I_{1}$. If $I \neq I_{1}$, then as in the proof of Lemma 2.3 we have $0<\mu\left(I_{1}\right)<1$ and $\mu\left(I_{1} \Delta t I_{1}\right)=0$ for any $t \in S$. These give that $\mu$ is not an extreme point of $M L(S)$. So $I$ must be a minimal finite left ideal. By the proof of the first part, $\mu$ is the arithmetic average on $I$.

COROLlaRy 3.3. For any left amenable semigroup $S, M L(S)$ has exposed points if and only if $S$ has finite left ideals. The number of exposed points of $M L(S)$ is exactly the number of minimal finite left ideals of $S$.

COROllary 3.4. If $S$ is a right cancellative, left amenable infinite semigroup, then $M L(S)$ has no exposed points.

Proof. For any $s \in S,|S s|=|S|$. So $S$ does not have finite left ideals.

Corollary 3.5. If $\operatorname{dim}(M L(S))<\infty$, then $S$ has finite left ideals.

Proof. If $\operatorname{dim}(M L(S))<\infty$, then $M L(S)$ is a compact convex subset of a Banach space. So it has exposed points.

COROLlary 3.6. Different exposed points of $M L(S)$ are linearly independent.

Corollary 3.3 extends [4], Corollary 4.1. Corollary 3.5 is the main result of Klawe [5].

Suppose $S$ is an infinite left amenable semigroup and $K$ is an invariant subset of $\beta S$. Let $M(S, K)$ denote the set of all $\mu \in M L(S)$ with its support contained in $K$ (see [1] for the definitions). Chou [1] proved that if $G$ is a countably infinite amenable group, then $M(G, K)$ 
has no exposed points. He asked whether this holds for any infinite amenable group. Our Corollary 3.4 gives a partial answer to this problem with $K=\beta G$.

Motivated by Granirer [3, Thm. 3.1], we get the following generalization.

TheOREM 3.7. If $M L(S)$ has exposed points, then it is the $w^{*}$-closed convex hull of all its exposed points.

Proof. Suppose $M L(S)$ has exposed points. Then $S$ has finite left ideals. Let $\left\{I_{\alpha}\right\}$ be the class of all its minimal finite left ideals and $A=\bigcup I_{\alpha}$. Then $A$ is a right ideal of $S$ since for any $s \in S, I_{\alpha} s$ is also a minimal left ideal. For any $\mu \in M L(S), \mu(A)=1$. Thus $\mu$ is the $w^{*}$-limit of a net $\left\{\mu_{\lambda}\right\}_{\lambda \in \Lambda}$ of finite means with supports in $A$. For each $\lambda \in \Lambda$, define

$$
\mu_{\lambda}^{\prime}=\sum_{\alpha} \mu_{\lambda}\left(I_{\alpha}\right) \varphi_{\alpha}
$$

where $\varphi_{\alpha}$ is the arithmetic average on $I_{\alpha}$. Then $\mu_{\lambda}^{\prime}$ is a convex combination of some $\varphi_{\alpha}$. Take a minimal finite left ideal $I_{0}=\left\{a_{1}, \ldots, a_{n}\right\}$. For any $I_{\alpha}$ and any $a \in I_{\alpha}$, it is easy to see that $\sum_{i=1}^{n} a_{i} \cdot \mu_{\lambda}(a)=\mu_{\lambda}\left(I_{\alpha}\right)$. So $\mu_{\lambda}^{\prime}=n^{-1} \sum_{i=1}^{n} a_{i} \cdot \mu_{\lambda}$. Since $\left\{\mu_{\lambda}\right\}$ converges to left invariance, we obtain that $\left\{\mu_{\lambda}^{\prime}\right\}$ converges to $\mu$ in the $w^{*}$-topology.

Corollary 3.8. (Granirer-Klawe Theorem. See [5].) For any left amenable semigroup $S, \operatorname{dim}(M L(S))=n$ if and only if $S$ contains exactly $n$ minimal finite left ideals.

Proof. If $S$ has $n$ minimal finite ideals, then $M L(S)$ has $n$ exposed points. By Corollary 3.6, $\operatorname{dim}(M L(S)) \geq n$. By Theorem 3.7, $M L(S)$ is the convex hull of those exposed points. $\operatorname{So} \operatorname{dim}(M L(S))=n$.

On the other hand, if $\operatorname{dim}(M L(S))=n$, by Corollary $3.5, S$ has finite left ideals. Again by Corollary 3.6, $S$ has only finitely many minimal finite left ideals. By the proof of the first part, this number is $n$.

\section{REFERENCES}

[1] C. Chou, On geometric property of the set of invariant means on a group, Proc. Amer. Math. Soc., 30 (1971), 296-302.

[2] M. M. Day, Amenable semigroups, Illinois J. Math., 1 (1957), 509-544. 
[3] E. E. Granirer, On amenable semigroups with a finite-dimensional set of invariant means I, Illinois J. Math., 7 (1963), 32-48.

[4] Exposed points of convex sets and weak sequential convergence, Mem. Amer. Math. Soc., 123 (1972).

[5] M. M. Klawe, On the dimension of left invariant means and left thick subsets, Trans. Amer. Math. Soc., 231 (1977), 507-518.

[6] T. Mitchell, Constant functions and left invariant means on semigroups, Trans. Amer. Math. Soc., 119 (1965), 244-261.

Received September 20,1985. This research was supported by the Killam Memorial Scholarship at the University of Alberta.

UNIVERSITY OF AlberTA

Edmonton, Alberta

Canada T6G 2G1 


\title{
PACIFIC JOURNAL OF MATHEMATICS \\ EDITORS
}

\author{
V. S. VARADARAJAN \\ (Managing Editor) \\ University of California \\ Los Angeles, CA 90024 \\ HERBERT CLEMENS \\ University of Utah \\ Salt Lake City, UT 84112 \\ R. FINN \\ Stanford University \\ Stanford, CA 94305
}

\author{
HERMANN FLASCHKA \\ University of Arizona \\ Tucson, AZ 85721
}

RAMESh A. GANGOLLI

University of Washington Seattle, WA 98195

VAUGHAN F. R. JONES

University of California

Berkeley, CA 94720

ROBION KIRBY

University of California

Berkeley, CA 94720
C. C. MOORE

University of California

Berkeley, CA 94720

H. SAMELSON

Stanford University

Stanford, CA 94305

HAROLD STARK

University of California, San Diego

La Jolla, CA 92093

\section{ASSOCIATE EDITORS}

\author{
R. ARENS \\ E. F. BECKENBACH \\ B. H. NeUmanN \\ F. WOLF \\ K. YOSHIDA \\ (1906-1982)

\section{SUPPORTING INSTITUTIONS} \\ UNIVERSITY OF ARIZONA \\ UNIVERSITY OF BRITISH COLUMBIA \\ CALIFORNIA INSTITUTE OF TECHNOLOGY \\ UNIVERSITY OF CALIFORNIA \\ MONTANA STATE UNIVERSITY \\ UNIVERSITY OF NEVADA, RENO \\ NEW MEXICO STATE UNIVERSITY \\ OREGON STATE UNIVERSITY \\ UNIVERSITY OF OREGON \\ UNIVERSITY OF SOUTHERN CALIFORNIA \\ STANFORD UNIVERSITY \\ UNIVERSITY OF HAWAII \\ UNIVERSITY OF TOKYO \\ UNIVERSITY OF UTAH \\ WASHINGTON STATE UNIVERSITY \\ UNIVERSITY OF WASHINGTON
}

The Supporting Institutions listed above contribute to the cost of publication of this Journal, but they are not owners or publishers and have no responsibility for its content or policies.

Mathematical papers intended for publication in the Pacific Journal of Mathematics should be in typed form or offset-reproduced (not dittoed), double spaced with large margins. Please do not use built up fractions in the text of the manuscript. However, you may use them in the displayed equations. Underline Greek letters in red, German in green, and script in blue. The first paragraph must be capable of being used separately as a synopsis of the entire paper. In particular it should contain no bibliographic references. Please propose a heading for the odd numbered pages of less than 35 characters. Manuscripts, in triplicate, may be sent to any one of the editors. Please classify according to the scheme of Math. Reviews, Index to Vol. 39. Supply name and address of author to whom proofs should be sent. All other communications should be addressed to the managing editor, or Elaine Barth, University of California, Los Angeles, California 90024.

There are page-charges associated with articles appearing in the Pacific Journal of Mathematics. These charges are expected to be paid by the author's University, Government Agency or Company. If the author or authors do not have access to such Institutional support these charges are waived. Single authors will receive 50 free reprints; joint authors will receive a total of 100 free reprints. Additional copies may be obtained at cost in multiples of 50 .

The Pacific Journal of Mathematics is issued monthly as of January 1966. Regular subscription rate: $\$ 190.00$ a year (5 Vols., 10 issues). Special rate: $\$ 95.00$ a year to individual members of supporting institutions.

Subscriptions, orders for numbers issued in the last three calendar years, and changes of address should be sent to Pacific Journal of Mathematics, P.O. Box 969, Carmel Valley, CA 93924, U.S.A. Old back numbers obtainable from Kraus Periodicals Co., Route 100, Millwood, NY 10546.

The Pacific Journal of Mathematics at P.O. Box 969, Carmel Valley, CA 93924 (ISSN 0030-8730) publish'es 5 volumes per year. Application to mail at Second-class postage rates is pending at Carmel Valley, California, and additional mailing offices. Postmaster: send address changes to Pacific Journal of Mathematics, P.O. Box 969, Carmel Valley, CA 93924.

PUBLISHED BY PACIFIC JOURNAL OF MATHEMATICS, A NON-PROFIT CORPORATION

Copyright (C) 1986 by Pacific Journal of Mathematics 


\section{Pacific Journal of Mathematics}

\section{Vol. 125, No. $2 \quad$ October, 1986}

Dale Edward Alspach, On $\mathscr{L}_{p, \lambda}$ spaces for small $\lambda \ldots \ldots \ldots \ldots \ldots \ldots 257$

Jong Sook Bae and Sangsuk Yie, Range of Gateaux differentiable operators

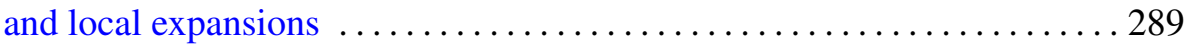

Hubert Berens and Lothar Hetzelt, On accretive operators on $l_{n}^{\infty} \ldots \ldots 301$

Marie-Françoise Bidaut-Véron, Global existence and uniqueness results for singular solutions of the capillarity equation ................. 317

Donald M. Davis and Mark Mahowald, Classification of the stable homotopy types of stunted real projective spaces ................. 335

Aad Dijksma, Heinz K. Langer and Hendrik S. V. de Snoo, Unitary

colligations in $\Pi_{\kappa}$-spaces, characteristic functions and Štraus extensions

Michel Enock and Jean-Marie Schwartz, Algèbres de Kac

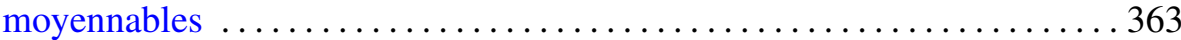

Seppo Granlund, Peter Lindqvist and Olli Martio, Note on the

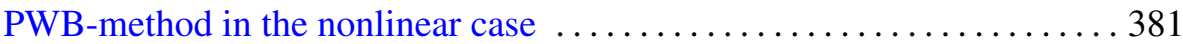

Palle E. T. Jorgensen, Analytic continuation of local representations of Lie

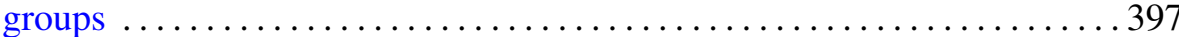

Robert P. Kaufman, Plane curves and removable sets ................409

José M. Montesinos and Wilbur Carrington Whitten, Constructions of two-fold branched covering spaces $\ldots \ldots \ldots \ldots \ldots$.

Benedict Seifert, Highly transitive group actions on trees and normalizing Tits systems

Charles Stuart Stanton, Counting functions and majorization for Jensen

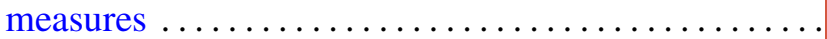

Luen-Fai Tam, On existence criteria for capillary free surfaces without gravity

Zhuocheng Yang, Exposed points of left invariant means 